\title{
Reviewer acknowledgement
}

Eric Arts $^{1 *}$ and Mark Boyd ${ }^{2}$

\section{Contributing reviewers}

AIDS Research and Therapy is very grateful for the generously-provided time and constructive, insightful reports provided by the journal's highly-qualified reviewers. We would like to show our appreciation by thanking the following people for their assistance with review of manuscripts for the journal in 2015.

$\begin{array}{lll}\text { Kevin Arien } & \text { Paul U. Cameron } & \text { Mark De Souza } \\ \text { Belgium } & \text { Australia } & \text { USA } \\ \text { Tristan Barber } & \text { Thomas Campbell } & \text { Greg Dekaban } \\ \text { UK } & \text { USA } & \text { Australia } \\ \text { Stephen Bar } & \text { Dianne Carey } & \text { Mark Douglas } \\ \text { Canada } & \text { Australia } & \text { Australia } \\ \text { Edward Berger } & \text { Kieran Cashin } & \text { Gwamaka Eliudi } \\ \text { USA } & \text { Australia } & \text { USA } \\ \text { Javier Bermudez-Silva } & \text { Derek Chan } & \text { Julian Elliot } \\ \text { Spain } & \text { Australia } & \text { Australia } \\ \text { Lionel Berthoux } & \text { Ploenchan Chetchotisakd } & \text { Jacqueline Flynn } \\ \text { Canada } & \text { Timor-Leste } & \text { Australia } \\ \text { Sanjay Bhagani } & \text { Ting Soo Chow } & \text { Nathan Ford } \\ \text { UK } & \text { Malaysia } & \text { Switzerland } \\ \text { Andrew Blance } & \text { David Clifford } & \text { Martyn French } \\ \text { Australia } & \text { USA } & \text { Australia } \\ \text { Mark Bloch } & \text { Eric Cohen } & \text { Yong Gao } \\ \text { Australia } & \text { Canada } & \text { USA } \\ \text { David Boettiger } & \text { Lois Conley } & \text { Roger Garsia } \\ \text { Australia } & \text { USA } & \text { Australia } \\ \text { Rohan Bopage } & \text { Suzanne Crowe } & \text { Davide Gibellini } \\ \text { Australia } & \text { Australia } & \text { Italy } \\ \text { Bruce Brew } & \text { Mark Danta } & \text { Charles Gilks } \\ \text { Australia } & \text { Australia } & \text { Australia } \\ & & \end{array}$

*Correspondence: aidsrestherapy@biomedcentral.com

1 Western University, London, Canada

Full list of author information is available at the end of the article

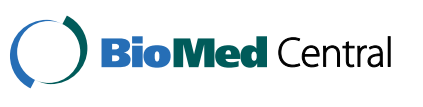

(c) 2016 Arts and Boyd. This article is distributed under the terms of the Creative Commons Attribution 4.0 International License (http://creativecommons.org/licenses/by/4.0/), which permits unrestricted use, distribution, and reproduction in any medium, provided you give appropriate credit to the original author(s) and the source, provide a link to the Creative Commons license, and indicate if changes were made. The Creative Commons Public Domain Dedication waiver (http://creativecommons.org/ publicdomain/zero/1.0/) applies to the data made available in this article, unless otherwise stated. 
Jonathan Golub

USA

David Gracey

Australia

Michael Grant

USA

Marguerite Guiguet

France

Manoji Gunathilake

Australia

Bridget Haire

Australia

Claudia Hawkins

USA

C. C. Hung

Taiwan

Awachana Jiamsakul

Australia

Chandy John

USA

Phyllis Kanki

USA

Richard Kaplan

South Africa

David Katzenstein

USA

Phillip Keen

Australia

Shane Kelly

Australia

Stephen Kerr

Thailand

Brian Kigozi

Uganda

Amelia Knopf

USA

Kersten Koelsch

Australia

John Koethe

USA

Scott Letendre

USA
Patrick Li

China

Michaela Lucas

Australia

Grace Lui

Hong Kong

Patrick Mallon

Ireland

Jamie Mann

Canada

Weerawat Manosuthi

Thailand

Louis Mansky

USA

Marianne Martinello

USA

Esteban Martinez

USA

Paul McKay

UK

James McMahon

Australia

Sanjay Mehta

Canada

D. Mohan

USA

Patricia Molina

USA

Martina Morris

USA

Marco Mura

Canada

Daniel Murray

Australia

Immaculate Nankya

Uganda

Mark Nelson

UK

Ichiro Nishimura

USA

David Nolan

Australia
Chidi Nwizu

USA

Phillipe Nyambi

USA

Catriona Ooi

Australia

Catherine Orrell

South Africa

C. Padmapriyadarsini

India

Alexander Pasternak

The Netherlands

Kathy Petoumenos

Australia

Chansavath Phetsouphanh

Australia

Angie Pinto

Australia

Jeffrey Post

Australia

Miguel Quinones-Mateu

USA

Roshan Ramlal

USA

Michael Roche

Australia

Jean-Pierre Routy

Canada

Allison Ruark

USA

Nathan Ryder

Australia

Carlos Seas

Peru

Leslie Shanks

Canada

Pasang Sherpa

USA

Tatsuo Shioda

Japan

Michael Silverman

Canada 
Don Smith

Australia

Kelli Snyders

Australia

Marcelo Soares

Brazil

Tim Spelman

Australia

Sanjay Swaminathan

Australia

Sue Swindells

USA

Alice Tang

USA
Denis Tebet

USA

Janine Trevillyan

Australia

Stuart Turville

Australia

ECM Van Gorp

The Netherlands

Johan Van Griensven

Belgium

Guido Vanham

Belgium

Angela Wahl

USA
Handan Wand

Australia

Alan Winston

UK

Ian Woolley

Australia

H. Manisha Yapa

Australia

Evy Yunihastuti

Indonesia

Yong-Tang Zheng

China

John Ziegler

Australia

Author details

${ }^{1}$ Western University, London, Canada. ${ }^{2}$ Kirby Institute

for Infection and Immunity in Society, Sydney, Australia.

Received: 19 February 2016 Accepted: 19 February 2016

Published online: 04 March 2016

Submit your next manuscript to BioMed Central and we will help you at every step:

- We accept pre-submission inquiries

- Our selector tool helps you to find the most relevant journal

- We provide round the clock customer support

- Convenient online submission

- Thorough peer review

- Inclusion in PubMed and all major indexing services

- Maximum visibility for your research

Submit your manuscript at www.biomedcentral.com/submit 